02

\title{
Влияние аккумуляционного слоя на спектральные свойства полностью покрытых майорановских нанопроводов
}

\author{
(C) A.A. Копасов ${ }^{1}$, А.С. Мельников ${ }^{1,2}$ \\ ${ }^{1}$ Институт фозики микроструктур РАН, \\ Нижний Новгород, Россия \\ ${ }^{2}$ Нижегородский государственный университет им. Н.И. Лобачевского, \\ Нижний Новгород, Россия \\ E-mail: kopasov@ipmras.ru
}

Поступила в Редакцию 26 марта 2020 г.

В окончательной редакции 26 марта 2020 г.

Принята к публикации 2 апреля 2020 г.

В рамках уравнений Боголюбова-де Жена исследовано влияние аккумуляционного слоя на спектральные свойства полупроводниковых нанопроводов, полностью покрытых сверхпроводящей оболочкой. Показано, что уменьшение длины аккумуляционного слоя, равно как и увеличение отношения Ферми скоростей в оболочке и в коре приводит к увеличению щели в спектре гибридного провода, тем самым, ограничивая область параметров, соответствующих топологически нетривиальной фазе. Наличие аккумуляционного слоя для квазичастиц на границе кор/оболочка также может приводить к возвратному поведению объемной щели в зависимости от внешнего магнитного потока. Такое возвратное поведение щели может наблюдаться экспериментально при измерениях периодичности тока через провод от напряжения на затворе в режиме кулоновской блокады.

Ключевые слова: полупроводниковые нанопровода, майорановские нанопровода, эффект близости, наведенная сверхпроводимость, связанные состояния в вихрях.

DOI: 10.21883/FTT.2020.09.49765.24H

\section{1. Введение}

Полупроводниковые нанопровода с сильным спинорбитальным взаимодействием, большим $g$-фактором и наведенной сверхпроводимостью являются перспективной платформой для поиска майорановских состояний в твердотельных системах [1-7]. Связано это с тем, что в таких нанопроводах в сильных магнитных полях $V_{z} \gg m^{*} \alpha^{2}$ (предел Китаева) может реализовываться так называемая бесспиновая $p$-волновая сверхпроводимость, возникновение которой должно сопровождаться появлением майорановских мод, локализованных на концах провода [8]. Здесь $V_{z}=\mu_{B} g H / 2$ - зеемановское расщепление, $\mu_{B}$ - магнетон Бора, $H-$ внешнее магнитное поле, направленное вдоль оси провода, $m^{*}-$ эффективйая масса электронов в полупроводнике, и $\alpha-$ константа спин-орбитального взаимодействия. Переход в топологическую фазу происходит с закрытием объемной щели в спектре квазичастичных возбуждений провода $E_{g}$ и последующим ее переоткрытием в топологически нетривиальной фазе $[9,10]$. Объемная щель $E_{g}$, защищающая майорановские состояния, пропорциональна параметру наведенной щели $\Delta_{i n d}$ и определяется прозрачностью границы между полупроводником (ПП) и сверхпроводником (СП). В последнее время был достигнут значительный прогресс в изготовлении гибридных структур из нанопроводов InAs, покрытых тонким слоем сверхпроводящего Al [11-14]. В частности, величина наведенной щели в таких структурах в нулевом магнитном поле $\Delta_{\text {ind }} \sim 0.2 \mathrm{meV}$ порядка сверхпроводящей щели в
Al $\left(\Delta_{\mathrm{Al}}=0.34 \mathrm{meV}\right)$, что свидетельствует о хорошей прозрачности границы ПП/СП. Наличие прозрачной границы ПП/СП также приводит к необходимости изучения эффектов, связанных с гибридизацией волновых функций в полупроводниковом коре и в металлической оболочке [15-18], и можно ожидать, что эффекты гибридизации должны быть чувствительны к возможным изгибам дна зоны проводимости в коре из-за контакта с металлом. Изгиб дна зоны проводимости в коре на границе с оболочкой естественным образом возникает в силу разных значений работ выхода в материалах и определяется параметром $W=E_{c}-E_{F}$, где $E_{c}-$ положение дна зоны проводимости в полупроводниковом коре на границе с металлом, и $E_{F}-$ энергия Ферми системы. Недавние экспериментальные измерения совместно с численными расчетами зонной структуры InAs/Al систем дают $W \approx-0.3 \mathrm{eV}$ [19], что свидетельствует в пользу наличия аккумуляционного слоя на границе ПП/СП (уровень Ферми располагается выше дна зоны проводимости в коре на границе кор/оболочка).

С другой стороны, основным недостатком текущего поколения майорановских нанопроводов на основе систем InAs/Al является большое значение приложенного магнитного поля для перехода в топологическую фазу $(H \sim 1 \mathrm{~T})$, которое, вообще говоря, сравнимо с критическим магнитным полем разрушения сверхпроводимости в оболочке [11-14]. Недавние работы [20-22] посвящены снятию данного ограничения. В частности, в работе [20] было предложено, что в полупроводниковых нанопроводах, полностью покрытых сверхпроводящей оболочкой, 
входящие в оболочку вихри могут перевести систему в топологическую фазу в магнитных полях $H \sim 0.1 \mathrm{~T}$. Основным требованием для реализации данного предложения является наличие пересечений подщелевых уровней уровня Ферми (наличие мод волноводного типа) и текстурированного спин-орбитального взаимодействия, определяемого радиальным электрическим полем на границе ПП/СП. В работе [22] было показано, что число таких пересечений определяется как числом поперечных мод в коре $\left(k_{F}^{c}\right)^{2} S$, так и конкуренцией между нормальным и андреевским отражением для квазичастиц как на границе ПП/СП, так и на внешней границе сверхпроводящей оболочки. Здесь $\hbar k_{F}^{c}-$ импульс Ферми в коре, и $S$ - площадь поперечного сечения кора. В свою очередь, наличие аккумуляционного слоя для квазичастиц вблизи границы ПП/СП должно приводить к перестройке спектра квазичастиц по сравнению со случаем плоского профиля потенциала, что несомненно должно отразиться на области параметров, при которых реализуется топологически нетривиальная фаза.

Принимая во внимание все вышесказанное, можно отметить, что исследование влияния аккумуляционного слоя на спектральные свойства полностью покрытых нанопроводов представляет интерес как в контексте их возможного использования для реализации майорановских мод, так и для объяснения недавних экспериментов [21]. Целью данной работы является исследование влияния аккумуляционного слоя на величину объемной щели в спектре полностью покрытых нанопроводов в зависимости от параметров слоя (длины слоя и отношения Ферми скоростей в оболочке и в коре) и приложенного магнитного потока без учета спиновых эффектов. Основным отличием данной работы от работы [22] является более детальный анализ влияния аккумуляционного слоя на спектры квазичастиц в рамках прямоугольного профиля потенциала для описания аккумуляционного слоя (в работе [22] был использован квадратичный профиль потенциала). В данной работе в рамках уравнений Боголюбова-де Жена для гибридной структуры показано, что уменьшение длины слоя (или увеличение отношения Ферми скоростей в оболочке и в коре) ведет к увеличению объемной щели в спектре нанопровода из-за уменьшения числа поперечных мод в коре. Таким образом, одно из влияний аккумуляционного слоя на спектр квазичастиц проявляется в уменьшении числа мод волноводного типа при уменьшении $\left(k_{F}^{c}\right)^{2} S$. Отсутствие волноводных мод в случае сильной аккумуляции квазичастиц вблизи границы ПП/СП без учета спиновых эффектов означает отсутствие топологических переходов в системе в случае слабого спин-орбитального взаимодействия [22]. Другое проявление аккумуляционного слоя состоит в возвратном поведении объемной щели в спектре нанопровода от приложенного магнитного потока из-за перестройки спектра квазичастиц и усиления роли орбитальных эффектов по сравнению со случаем плоского профиля дна зоны проводимости в коре. Такое возвратное поведение жесткой щели при изменении приложенного магнитного потока должно проявляться в особенностях транспорта в условиях кулоновской блокады.

\section{2. Метод}

В настоящей работе рассматривается полупроводниковый провод, полностью покрытый сверхпроводящей оболочкой, во внешнем магнитном поле $H$, направленном вдоль оси провода. Анализ основан на уравнениях Боголюбова-де Жена для гибридной структуры

$$
\begin{gathered}
H_{\mathrm{BdG}} \Psi(\mathbf{r})=E \Psi(\mathbf{r}), \\
H_{\mathrm{BdG}}=\tau_{z}\left\{\mathbf{P}\left[[2 m(\mathbf{r})]^{-1}\right] \mathbf{P}+U(\mathbf{r})\right\} \\
+\tau_{x} \operatorname{Re} \Delta(\mathbf{r})-\tau_{y} \operatorname{Im} \Delta(\mathbf{r}) .
\end{gathered}
$$

Здесь $\mathbf{P}=\left(\mathbf{p}+\tau_{z} e \mathbf{A} / c\right), m(\mathbf{r})-$ неоднородная эффективная масса, $U(\mathbf{r})-$ ограничивающий потенциал, $\tau_{i}$ $(i=x, y, z)$ - матрицы Паули в пространстве электрон-дырка, и сверхпроводящий параметр порядка $\Delta(\mathbf{r})$ отличен от нуля только в оболочке. Для простоты мы рассматриваем гибридный провод с круглым поперечным сечением [23] и используем цилиндрические координаты $\mathbf{r}=(r, \varphi, z)$. Пренебрегая неоднородностью параметра порядка в оболочке, мы используем следующее упрощенное выражение, описывающее переключения между различными вихревыми состояниями при изменении магнитного потока

$$
\Delta=\Delta_{0}\left[1-\gamma\left(\Phi / \Phi_{0}-n\right)^{2}\right] \exp (-i n \varphi),
$$

где $\Delta_{0}-$ сверхпроводящая щель в оболочке при $H=0$, $n(\Phi)=\left\lfloor 1 / 2+\Phi / \Phi_{0}\right\rfloor,\lfloor x\rfloor$ - целая часть, $\gamma \sim \xi_{s}^{2} / R_{c}^{2}$ для толщин оболочки $d_{s} \ll R_{c}, \xi_{s}-$ сверхпроводящая длина когерентности, $\Phi=\pi H R_{c}^{2}$ и $\Phi_{0}=\pi \hbar c / e-$ магнитный поток и квант магнитного потока, соответственно. Более строгий анализ спектральных свойств полностью покрытых нанопроводов должен проводиться с использованием точного профиля сверхпроводящего параметра порядка $\Delta(\mathbf{r})$, определяемого из решения уравнения самосогласования [24-26]. Выбирая калибровку векторного потенциала в виде $A_{\varphi}=H r / 2, A_{r}=A_{z}=0$, ищем решения уравнений (1) в виде

$$
\Psi(\mathbf{r})=\exp \left(i k_{z} z+i \mu \varphi-i \tau_{z} n \varphi / 2\right) \Psi_{\mu, k_{z}}(r),
$$

где $\mu$ принимает целые (полуцелые) значения для четных (нечетных) $n$. Подставляя выражение (3) в уравнения (1) в полупроводниковом коре, мы получаем следующую задачу на собственные значения

$$
\begin{gathered}
\mathscr{L} \Psi_{\mu, k_{z}}(r)=E \Psi_{\mu, k_{z}}(r), \\
\mathscr{L}=\tau_{z} \frac{\hbar^{2}}{2 m}\left\{-\frac{1}{r} \frac{d}{d r}\left(r \frac{d}{d r}\right)\right. \\
\left.+\left[\frac{\mu}{r}+\tau_{z} \frac{m^{*}}{\hbar} v(r)\right]^{2}+k_{z}^{2}\right\}+\tau_{z} U(r) .
\end{gathered}
$$

Здесь $v(r)=\left(\hbar / 2 m^{*} r\right)\left(\pi H r^{2} / \Phi_{0}-n\right)$. Спектр квазичастиц в гибридном проводе может быть найден при помощи общих решений радиальных уравнений (4)

$$
f_{+}(r)=C_{+} J_{\left|\mu_{e}\right|}\left(\kappa_{e} r\right)+B_{+} Y_{\left|\mu_{e}\right|}\left(\kappa_{e} r\right),
$$



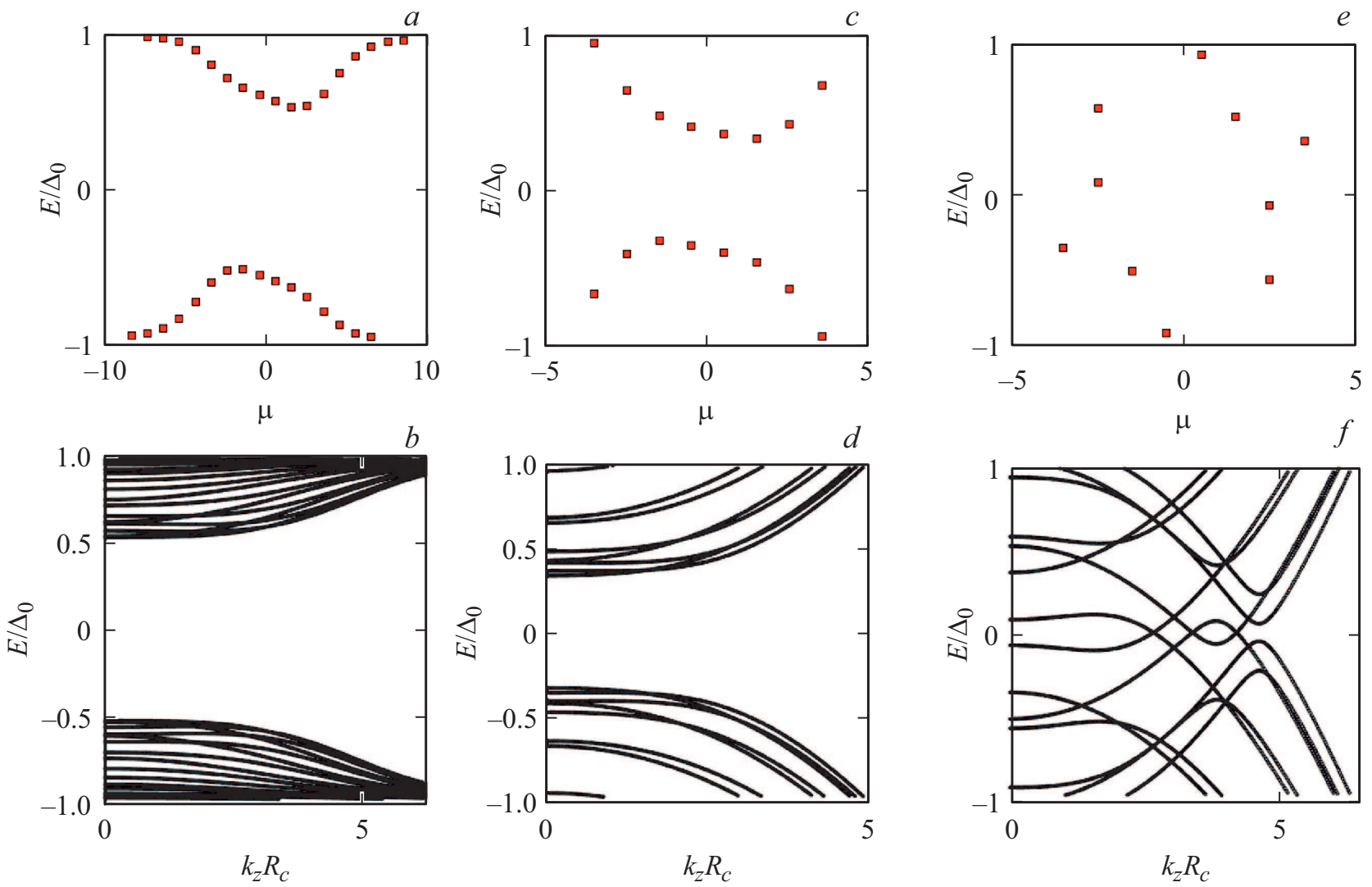

Рис. 1. $(a, c)$ - Зависимости $E(\mu)$ при $k_{z}=0$ в момент входа вихря $\Phi / \Phi_{0}=0.5$ для $R_{c}=1.2 \xi_{s}, \xi_{s}=\hbar v_{F}^{s} / \Delta_{0}, \gamma=0.1, L / R_{c}=0.5$, $\eta=1.2, d_{s} \gtrsim \xi_{s}(a)$ и $d_{s}=0.5 R_{c}(c)$. $(b, d)$ - Типичные спектры квазичастиц $E\left(k_{z}\right)$ для параметров, соответствующих панелям $(a)$ и $(c)$. На панелях $(e)$ и $(f)$ показаны зависимости $E(\mu)$ при $k_{z}=0$ и спектры квазичастиц $E\left(k_{z}\right)$, соответственно, для $\Phi / \Phi_{0}=0.5$, $R_{c}=1.2 \xi_{s}, \eta=1, L / R_{c}=0.8, d_{s} / R_{c}=0.3$ и $\gamma=0.1$.

$$
\begin{gathered}
f_{-}(r)=C_{-} J_{\left|\mu_{h}\right|}\left(\kappa_{h} r\right)+B_{-} Y_{\left|\mu_{h}\right|}\left(\kappa_{h} r\right), \\
\kappa_{e}^{2}=\left(2 m^{*} / \hbar^{2}\right)\left(E_{\perp}+E-\mu_{e} \hbar \omega_{c} / 2\right), \\
\kappa_{h}^{2}=\left(2 m^{*} / \hbar^{2}\right)\left(E_{\perp}-E+\mu_{h} \hbar \omega_{c} / 2\right)
\end{gathered}
$$

и граничных условий. Здесь $\Psi_{\mu, k_{z}}(r)=\left[f_{+}(r), f_{-}(r)\right]^{T}$, $C_{ \pm}$и $B_{ \pm}$- произвольные постоянные, $J_{p}(x)$ и $Y_{p}(x)-$ функции Бесселя первого и второго рода порядка $p$, соответственно, $\mu_{e, h}=\mu \pm n / 2, E_{\perp}^{c}=\left(E_{F}^{c}-\hbar^{2} k_{z}^{2} / 2 m^{*}\right)$ (мы считаем $U=-E_{F}^{c}$ постоянным в пределах аккумуляционного слоя), $E_{F}^{c}-$ энергия Ферми в коре, и $\omega_{c}=e H / m^{*} c-$ циклотронная частота. Решения вида (5) справедливы в случае оносительно слабых магнитных полей $\Phi \lesssim \Phi_{0}$. Для простоты наличие аккумуляционного слоя для квазичастиц вблизи границы СП/ПП длины $L$ описывается с помощью жестких граничных условий на радиальные части волновых функций $\Psi_{\mu, k_{z}}\left(R_{c}-L\right)=0$, а граница ПП/СП описывается с помощью граничных условий, учитывающих конкуренцию между андреевским и нормальным отражением (вывод граничных условий на границе кор/оболочка представлен в работе [22]). Во всех численных расчетах мы выбираем $\Delta_{0} / E_{F}^{s}=0.01$, где $E_{F}^{s}-$ энергия Ферми в оболочке. Мы выражаем число поперечных мод в коре

$$
N_{t} \approx\left(k_{F}^{c}\right)^{2} S=\left(m^{*} / m_{s}\right)^{2}\left(k_{F}^{s} R_{c}\right)^{2}\left(\pi L / \eta^{2} R_{c}\right)\left(2-L / R_{c}\right)
$$

через отношение $L / R_{c}$ и отношение Ферми скоростей в оболочке и в коре $\eta=m^{*} k_{F}^{s} / m_{s} k_{F}^{c}$, где $\hbar k_{F}^{s}-$ импульс Ферми в оболочке. Мы также выбираем отношение эффективных масс в коре и в оболочке в соответствии со свойствами InAs/Al нанопроводов [27] $m^{*} / m_{s}=0.026$.

\section{3. Зависимости объемной щели в спектре гибридного нанопровода от параметров аккумуляционного слоя}

Вначале рассмотрим, как происходит перестройка спектра подщелевых состояний в полностью покрытых нанопроводах из-за аккумуляционного слоя. В данном разделе для простоты мы будем рассматривать одноквантовый вихрь $n=1$. По аналогии с вихрем в $s$-волновом сверхпроводнике $[28,29]$ в случае доминирующего андреевского рассеяния на границе ПП/СП для плоского профиля потенциала в коре образуются связанные состояния Кароли-де Жена-Матрикона (КдЖМ) [22], и связанным состояниям с низшей энергией соответствуют наименьшие $z$-проекции углового момента $\mu= \pm 1 / 2$. Данная простая картина, конечно, меняется при учете нормального отражения от границы ПП/СП и от внеш- 
ней границы сверхпроводящей оболочки. В согласии с предыдущими теоретическими работами, посвященными изучению спектральных свойств вихрей в мезоскопических сверхпроводниках [30-32], наличие нормального отражения в полностью покрытых нанопроводах также приводит к осцилляциям подщелевых уровней и к появлению мод волноводного типа за счет интерференционных эффектов [22].

Типичные спектры квазичастиц в полностью покрытых нанопроводах при наличии аккумуляционного слоя представлены на рис. 1. Из рис. 1 можно видеть, что наличие аккумуляционного слоя для квазичастиц вблизи границы ПП/СП длины $L$ приводит к транформации зависимостей $E(\mu)$ при фиксированном $k_{z}$ аналогично вихрю, запиннингованном на колумнарном дефекте [33]. В частности, зависимости $|E(\mu)|$ при фиксированном $k_{z}$ имеют минимум при $\mu \approx \pm \mu_{c}, \mu_{c}=k_{\perp}^{c}\left(R_{c}-L\right)$, а энергии связанных состояний при уменьшении $|\mu|<\mu_{c}$ должны приближаться к наведенной щели за счет нормального отражения для квазичастиц от внутренней границы аккумуляционного слоя при $r=\left(R_{c}-L\right)$. Естественно ожидать, что вышеописанная трансформация спектров квазичастиц из-за аккумуляционного слоя приведет к усилению роли орбитальных эффектов, так как допплеровский сдвиг подщелевых уровней $\delta E_{\mu} \propto \mu H$ [34]. Панели $(b)$ и $(d)$ на рис. 1 показывают, что учет нормального отражения для квазичастиц от внешней границы сверхпроводящей оболочки на квазичастичный спектр приводит к уменьшению объемной щели в системе из-за осцилляций подщелевых уровней. Наконец, на панелях $(e, f)$ на рис. 1 показано как влияние перестройки спектра из-за аккумуляционного слоя [32] (e), так и появление пересечений подщелевыми уровнями уровня Ферми из-за конкуренции нормального и андреевского рассеяния [30-32] $(f)$.

Обсудим влияние аккумуляционного слоя на возможность появления майорановких мод на краях полностью покрытых нанопроводов при учете текстурированного взаимодействия. В силу того обстоятельства, что спин-орбитальное взаимодействие Рашбы с радиальным вектором нормали приводит к гибридизации вихревых состояний с отличающимися на единицу $z$-проекциями углового момента, майорановские состояния в многомодовых проводах должны возникать в области параметров, соответствующих нечетному числу пересечений невозмущенных уровней с $\mu= \pm 1 / 2$ уровня Ферми (без учета спин-орбитального взаимодействия) [22]. Однако, как показано на рис. 1, перестройка спектра квазичастиц из-за аккумуляционного слоя приводит к увеличению энергии связанных состояний с $\mu= \pm 1 / 2$ и, соответственно, к отсутствию требуемых пересечений. Таким образом, можно сделать вывод, что формирование аккумуляционного слоя в полупроводниковом коре нежелательно в контексте реализации майорановских мод в таких системах.

Зависимости объемной щели в спектре полностью покрытого нанопровода от параметров аккумуляционного слоя показаны на рис. 2 и 3. На рис. 2 представлены зависимости щели от длины аккумуляционного

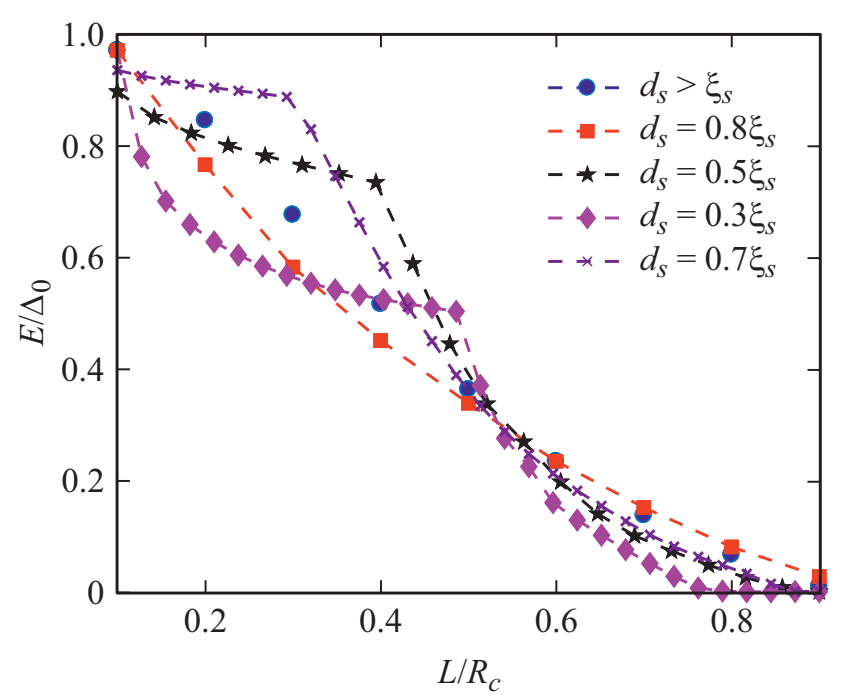

Рис. 2. Типичные зависимости объемной щели в спектре нанопровода $E_{g}$ в момент входа вихря $\Phi / \Phi_{0}=0.5$ от длины аккумуляционного слоя $L$ для $R_{c}=1.5 \xi_{s}, \gamma=0.1, \eta=1$ при различных значениях толщины сверхпроводящей оболочки $d_{s}$.

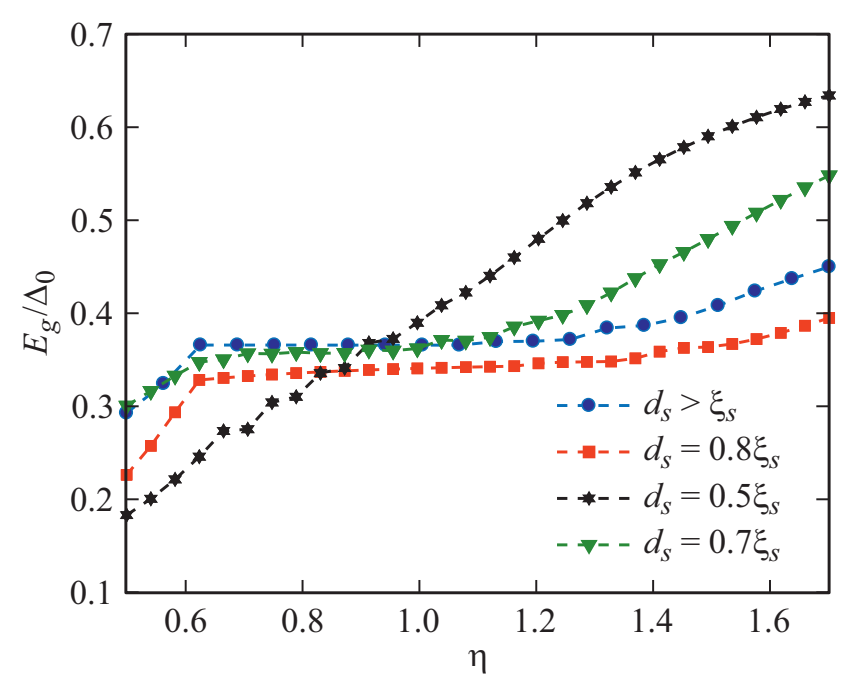

Рис. 3. Типичные зависимости $E_{g}(\eta)$ для $\Phi / \Phi_{0}=0.5$, $R_{c}=1.5 \xi_{s}, L / R_{c}=0.5, \gamma=0.1$ при различных значениях $d_{s}$.

слоя при фиксированном отношении Ферми скоростей в оболочке и в коре. Исходя из аналогии с одноквантовым вихрем в $s$-волновом сверхпроводнике, запиннингованном на колумнарном дефекте, наивно можно было бы ожидать линейную зависимость щели от длины аккумуляционного слоя $E_{g}=|\Delta(\Phi)|\left(1-L / R_{c}\right)$. Такое поведение получается в предположении, что щель в спектре определяется следующим выражением $E^{(0)}\left(\mu_{c}\right)$, где $E^{(0)}(\mu)=|\Delta(\Phi)| / k_{\perp}^{c} R_{c}$ - спектр Кароли-де ЖенаМатрикона в случае плоского профиля потенциала в коре [22]. Здесь $k_{\perp}^{c}=\left[\left(k_{F}^{c}\right)^{2}-k_{z}^{2}\right]^{1 / 2}$. Однако из рис. 2 видно, что зависимости $E_{g}(L)$ не являются линейными, что, по-видимому, вызвано наличием нормального рассеяния на границе кор/оболочка и на внешней границе 
гибридной структуры, а также неквазиклассическими поправками к спектру при малых $L$ в случае $\left(k_{F}^{c}\right)^{2} S \sim 1$. На рис. 3 изображены типичные зависимости $E_{g}(\eta)$ при фиксированной длине аккумуляционного слоя. На рис. 3 явно наблюдается увеличение щели в спектре нанопровода при увеличении отношения Ферми скоростей в оболочке и в коре из-за уменьшения числа поперечных мод в коре.

Важно отметить, что величина объемной щели в спектре $E_{g}$ при изучении транспорта через провод в режиме кулоновской блокады в действительности контролирует периодичность тока через провод в зависимости от напряжения на затворе $[35,36]$. В силу достаточно сильной зависимости объемной щели в спектре гибридного провода от параметров аккумуляционного слоя (см. рис. 2 и 3), параметры слоя (характерный пространственный масштаб и число поперечных мод) могут быть оценены из измерений периодичности тока через провод в зависимости от напряжения на затворе.

\section{4. Возвратное поведение щели в спектре нанопровода при изменении магнитного потока}

Теперь рассмотрим влияние аккумуляционного слоя на зависимости объемной щели в спектре провода от внешнего магнитного потока $E_{g}(\Phi)$. Типичные зависимости $E_{g}(\Phi)$ представлены на рис. 4. Из рис. 4 можно видеть скачкообразные подавления объемной щели в момент входа вихрей при полуцелых $\Phi / \Phi_{0}$. Связано это с тем, что при переключении завихренности сверхпроводящего параметра порядка $n$ взаимодействуют электронные и дырочные состояния с разными $z$-проекциями углового момента $(\mu-n / 2)$ и $(\mu+n / 2)$ соответственно. Из рис. 4 также видно, что подавление жесткой щели при входе вихрей и допплеровские сдвиги

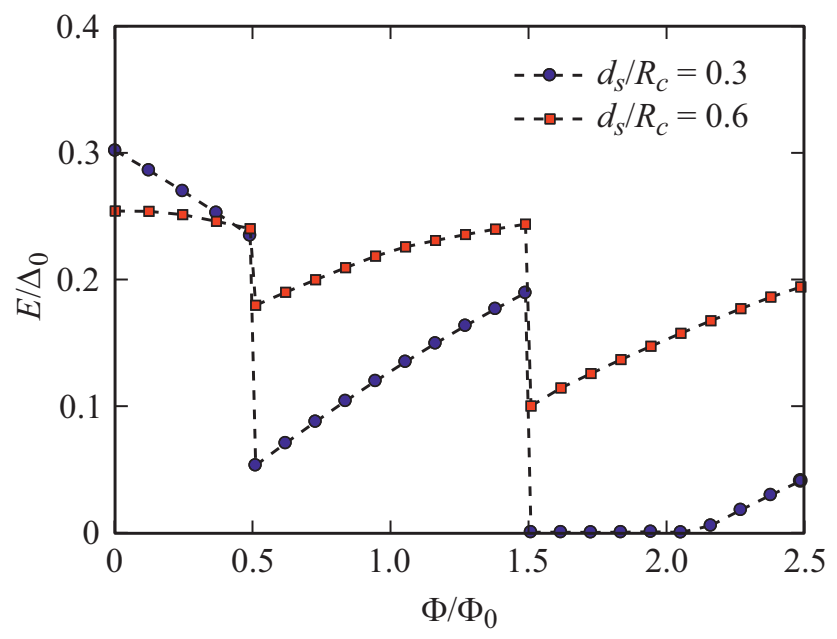

Рис. 4. Зависимость щели в спектре гибридного нанопровода в зависимости от внешнего магнитного потока для $R_{c}=1.5 \xi_{s}$, $L / R_{c}=0.6, \gamma=0.1, \eta=0.8$ при различных значениях $d_{s}$. подщелевых уровней при изменении магнитного поля могут приводить к возвратному поведению $E_{g}(\Phi)$. Такое необычное поведение объемной щели при изменении магнитного потока, вызванное вышеописанной трансформацией спектра квазичастиц, должно проявляться в переключениях периодичности тока через провод в зависимости от напряжения на затворе при изменении внешнего магнитного потока в условиях кулоновской блокады. В частности, скачкообразное подавление щели при входе вихря может приводить к резкому переключению периодичности тока с $2 e$ на $e$ при входе вихря и к последующему восстановлению к $2 e$ периодичности при увеличении магнитного потока в пределах заданного вихревого состояния. Рассматривая одноквантовый вихрь для примера и выбирая типичное значение $E_{g}=0.25 \Delta_{0}$ для сверхпроводящей оболочки c $\Delta_{0} \approx 200 \mu \mathrm{eV}$, можно оценить соответствующий диапазон температур, при котором должен наблюдаться эффект четности $T \lesssim T^{*}=E_{g} / \ln \left(N_{\text {eff }}\right) \approx 0.6 \mathrm{~K}$ [35-36].

\section{5. Заключение}

В настоящей работе в рамках уравнений Боголюбоваде Жена изучено влияние аккумуляционного слоя на спектральные свойства полупроводниковых нанопроводов, полностью покрытых сверхпроводящей оболочкой. В работе показано, что уменьшение длины аккумуляционного слоя $L$ и увеличение отношения Ферми скоростей в оболочке и в коре приводят к увеличению щели в спектре гибридного провода. Таким образом, наличие аккумуляционного слоя в коре вносит дополнительные ограничения на область параметров системы, при которых возможна реализация топологически нетривиальной фазы. Наличие аккумуляционного слоя для квазичастиц вблизи границы ПП/СП также может приводить к возвратному поведению объемной щели в спектре нанопровода в зависимости от внешнего магнитного потока из-за трансформации спектра квазичастиц и связанного с ней усиления орбитальных эффектов. Возвратное поведение щели от магнитного потока может наблюдаться экспериментально в измерениях периодичности тока через провод в зависимости от напряжения на затворе в условиях кулоновской блокады.

\section{Финансирование работы}

Работа выполнена при поддержке гранта Российского фонда фундаментальных исследований (гранты № 17-52-12044 и № 18-02-00390), госзадания ИФМ РАН № 0035-2019-0021 (раздел 3), и гранта Российского научного фонда № 20-12-00053 (раздел 4).

\section{Конфликт интересов}

Авторы заявляют, что у них нет конфликта интересов. 


\section{Список литературы}

[1] A.Yu. Kitaev. Phys. Usp. 44, 131 (2001).

[2] C. Nayak, S.H. Simon, A. Stern, M. Freedman, S. Das Sarma. Rev. Mod. Phys. 80, 1083 (2008).

[3] J. Alicea, Y. Oreg, G. Refael, F. von Oppen, M.P.A. Fisher. Nature Phys. 7, 412 (2011).

[4] D. Aasen, M. Hell, R. V. Mishmash, A. Higginbotham, J. Danon, M. Leijnse, T.S. Jespersen, J.A. Folk, C.M. Marcus, K. Flensberg, J. Alicea. Phys. Rev. X 6, 031016 (2016).

[5] J. Alicea. Rep. Prog. Phys. 75, 076501 (2012).

[6] S.R. Elliott, M. Franz. Rev. Mod. Phys. 87, 137 (2015).

[7] R. Aguado, Riv. Nuovo Cimento Soc. Ital. Fis. 40, 523 (2017).

[8] F. von Oppen, Y. Peng, F. Pientka. Topological superconducting phases in one dimension. Oxford University Press (2017).

[9] R.M. Lutchyn, J.D. Sau, S.Das Sarma. Phys. Rev. Lett. 105, 077001 (2010).

[10] Y. Oreg, G. Refael, F. von Oppen. Phys. Rev. Lett. 105, 177002 (2010).

[11] W. Chang, S.M. Albrecht, T.S. Jespersen, F. Kuemmeth, P. Krogstrup, J. Nygard, C.M. Marcus. Nature Nanotechnol. 10, 232 (2015).

[12] A.P. Higginbotham, S.M. Albrecht, G. Kirsanskas, W. Chang, F. Kuemmeth, P. Krogstrup, T.S. Jespersen, J. Nygard, K. Flensberg, C.M. Marcus. Nature Phys. 11, 1017 (2015).

[13] P. Krogstrup, N.L.B. Ziino, W. Chang, S.M. Albrecht, M.H. Madsen, E. Johnson, J. Nygard, C.M. Marcus, T.S. Jespersen. Nature Mater. 14, 400 (2015).

[14] S.M. Albrecht, A.P. Higginbotham, M. Madsen, F. Kuemmeth, T.S. Jespersen, J. Nygard, P. Krogstrup, C.M. Marcus. Nature 531, 206 (2016).

[15] V.E. Degtyarev, S.V. Khazanova, N.V. Demarina. Sci. Rep. 7, 3411 (2017).

[16] A.E. Antipov, A. Bargerbos, G.W. Winkler, B. Bauer, E. Rossi, R.M. Lutchyn. Phys. Rev. X 8, 031041 (2018).

[17] A.E.G. Mikkelsen, P. Kotetes, P. Krogstrup, K. Flensberg. Phys. Rev. X 8, 031040 (2018).

[18] B.D. Woods, T.D. Stanescu, S. Das Sarma. Phys. Rev. B 98, 035428 (2018).

[19] S. Schuwalow, N.B.M. Schroeter, J. Gukelberger, C. Thomas, V. Strocov, J. Gamble, A. Chikina, M. Caputo, J. Krieger, G.C. Gardner, M. Troyer, G. Aeppli, M.J. Manfra, P. Krogstrup. arXiv:1910:02735 (2019).

[20] R.M. Lutchyn, G.W. Winkler, B. van Heck, T. Karzig, K. Flensberg, L.I. Glazman, C. Nayak. arXiv:1809.05512 (2018).

[21] S. Vaitiekenas, M.-T. Deng, P. Krogstrup, C.M. Marcus. arXiv:1809.05513 (2018).

[22] A.A. Kopasov, A.S. Mel'nikov. Phys. Rev. B 101, 054515 (2020).

[23] N. Luo, G. Liao, H.Q. Xu. AIP Advances 6, 125109 (2016).

[24] S.M.M. Virtanen, M.M. Salomaa. Phys. Rev. B 60, 14581 (1999).

[25] K. Tanaka, I. Robel, B. Janko. Proc. Natl. Acad. Sci. USA 99, 5233 (2002).

[26] L.F. Zhang, L. Covaci, M.V. Milosevic, G.R. Berdiyorov, F.M. Peeters. Phys. Rev. Lett 109, 107001 (2012).

[27] I. Vurgaftman, J.R. Meyer, L.R. Ram-Mohan. J. Appl. Phys. 89, 5815 (2001).

[28] C. Caroli, P.-G. de Gennes, J. Matricon. Phys. Lett. 9, 307 (1964).
[29] J. Bardeen, R. Kummel, A.E. Jacobs, L. Tewordt. Phys. Rev. 187, 556 (1969).

[30] N.B. Kopnin, A.S. Mel'nikov, V.I. Pozdnyakova, D.A. Ryzhov, I.A. Shereshevskii, V.M. Vinokur. Phys. Rev. Lett. 95, 197002 (2005).

[31] N.B. Kopnin, A.S. Mel'nikov, V.I. Pozdnyakova, D.A. Ryzhov, I.A. Shereshevskii, V.M. Vinokur. Phys. Rev. B 75, 024514 (2007).

[32] A.S. Mel'nikov, D.A. Ryzhov, M.A. Silaev. Phys. Rev. B 79, 134521 (2009)

[33] A.S. Mel'nikov, A.V. Samokhvalov, M.N. Zubarev. Phys. Rev. B 79, 134529 (2009).

[34] B.E. Hansen. Phys. Lett. 27A, 576 (1968).

[35] D.V. Averin, Yu.V. Nazarov. Phys. Rev. Lett. 69, 1993 (1992).

[36] M.T. Tuominen, J.M. Hergenrother, T.S. Tighe, M. Tinkham. Phys. Rev. Lett. 69, 1997 (1992).

Редактор Т.Н. Василевская 